\title{
STAT3 regulates arginase-I in myeloid-derived suppressor cells from cancer patients
} David Vasquez-Dunddel, ${ }^{1}$ Fan Pan, ${ }^{2}$ Qi Zeng, ${ }^{1}$ Mikhail Gorbounov, ${ }^{1}$ Emilia Albesiano, ${ }^{2}$ Juan Fu, ${ }^{1,2}$
Richard L. Blosser, ${ }^{2}$ Ada J. Tam, ${ }^{2}$ Tullia Bruno, ${ }^{3}$ Hao Zhang, ${ }^{4}$ Drew Pardoll,,2 and Young Kim ${ }^{1,2}$

1Department of Otolaryngology — Head and Neck Surgery and 2 Immunology and Hematopoiesis Division, Sidney Kimmel Comprehensive Cancer Center, Johns Hopkins University, School of Medicine, Baltimore, Maryland, USA. 3University of Colorado, School of Medicine, Integrated Department of Immunology, Denver, Colorado, USA. ${ }^{4}$ Cell Cytometry Core, Johns Hopkins University, School of Public Health, Baltimore, Maryland, USA.

\begin{abstract}
Myeloid-derived suppressor cells (MDSC) play a key immunosuppressive role in various types of cancer, including head and neck squamous cell carcinoma (HNSCC). In this study, we characterized CD14 ${ }^{+} \mathrm{HLA}_{-}-\mathrm{DR} \mathrm{R}^{-/ 1 \mathrm{l}}$ cells sorted from the tumors, draining lymph nodes, and peripheral blood of HNSCC patients. CD14 HLA$\mathrm{DR}^{-/ 10}$ cells were phenotyped as $\mathrm{CD} 1 \mathrm{~b}^{+}, \mathrm{CD}^{+}, \mathrm{CD}^{+} 4^{+}$, arginase- $\mathrm{I}^{+}$, and $\mathrm{ROS}^{+}$. In all 3 compartments, they suppressed autologous, antigen-independent $T$ cell proliferation in a differential manner. The abundance of MDSC correlated with stage, but did not correlate with previous treatment with radiation or subsites of HNSCC. Interestingly, MDSC from all 3 compartments showed high phosphorylated STAT3 levels that correlated with arginase-I expression levels and activity. Stattic, a STAT3-specific inhibitor, and STAT3-targeted siRNA abrogated MDSC's suppressive function. Inhibition of STAT3 signaling also resulted in decreased arginase-I activity. Analysis of the human arginase-I promoter region showed multiple STAT3-binding elements, and ChIP demonstrated that phosphorylated STAT3 binds to multiple sites in the arginase-I promoter. Finally, rescue of arginase-I activity after STAT3 blockade restored MDSC's suppressive function. Taken together, these results demonstrate that the suppressive function of arginase-I in both infiltrating and circulating MDSC is a downstream target of activated STAT3.
\end{abstract}

\section{Introduction}

The heterogeneous myeloid-derived suppressor cells (MDSC) play an immune-suppressive role in tumor-bearing animals as well as in the peripheral blood (PB) of cancer patients with various types of malignancies (1-3). CD34 MDSC were first isolated from head and neck squamous cell carcinoma (HNSCC) patients due to their high abundance in this tumor (4). Clinical correlation studies in breast, colorectal, pancreatic, esophageal, and gastric cancer patients demonstrated that increased MDSC levels may be an important independent prognostic factor for survival $(5,6)$. For lung cancer patients, MDSC level is negatively correlated with responsiveness to standard chemotherapy (7). In general, MDSC from cancer patients express the common myeloid marker CD33 and CD11b, but lack mature myeloid or lymphoid markers such as HLA-DR $(8,9)$. In mice, these cells have been subdivided into granulocytic $\left(\mathrm{CD} 11 \mathrm{~b}^{+} \mathrm{Ly} 6 \mathrm{G}^{+} \mathrm{Ly} 6 \mathrm{C}^{\mathrm{lo}}\right)$ or monocytic (CD11b ${ }^{+}$Ly6G-Ly6C hi) populations (10). Among cancer patients, it has been proposed that monocytic MDSC tend to be $\mathrm{CD}_{1} 4^{+}$, while the granulocytic MDSC are $\mathrm{CD} 15^{+}$, but the functional significance of these phenotypic categorizations in the human system is still unclear $(11,12)$. Mandruzzato et al. studied both monocytic and granulocytic MDSC from PB of colon cancer and melanoma patients and found a correlation between the expression of IL-4R and suppressive activity in the monocytic population. But this study also showed that the CD14 and CD15 populations overlapped significantly (13).

In terms of established molecular mechanisms of MDSC's suppressive function, some of the downstream mediators have been characterized from tumor bearing mice. Depletion of L-arginine

Conflict of interest: The authors have declared that no conflict of interest exists. Citation for this article: J Clin Invest. 2013;123(4):1580-1589. doi:10.1172/JCI60083.
(L-arg) and cysteine, increased nitric oxide (NO), and upregulation of ROS, peroxynitrates, and multiple cytokines appear to mediate MDSC's T cell-suppressive function (14-17). However, the upstream regulators of these suppressive mediators have not been clearly delineated, particularly from cancer patients. In this regard, several reports that focused on MDSC from cancer patients noted the importance of STAT3 signaling in these cells $(18,19)$. However, how STAT3 regulates downstream mediators in MDSC from human cancer patients is not clear.

Marigo et al. showed that $\mathrm{C} / \mathrm{EBP} \beta$ transcription factor in the myeloid compartment is critical in regulating immunosuppression (20), and Zhang et al. showed that STAT3 directly controls G-CSF-dependent expression of C/EBP $\beta$ in emergency granulopoiesis (21). C/EBP $\beta$ has been shown to regulate arginase-I (ARG1) in murine macrophages (22). In other murine studies, inhibition of STAT3 signaling in the myeloid compartment induced an antitumor response (23). STAT3-dependent expansion and differentiation of MDSC has been proposed to occur through the regulation of NADH oxidase $(24,25)$. Whether STAT3 directly controls other key downstream mediators of MDSC function is unknown. STAT1 and STAT6 as well as NF-K $\beta$ have been reported to increase ARG1 and iNOS activity in MDSC in several murine models (26-28). In murine inflammatory models, STAT3 was found to regulate ARG1 in mycobacteria-infected macrophages (29). However, whether these STAT signaling pathways in murine MDSC are also applicable in MDSC from cancer patients is still unclear (30).

Furthermore, although MDSC from the tumor and the periphery appear to have differential function in mice, there are no comparable studies in the human system. Moreover, it is unclear whether STAT3 signaling is important in the tumor microenvironment in comparison with the periphery in the human system (31). The current understanding of human MDSC is primarily derived from $\mathrm{PB}$, 

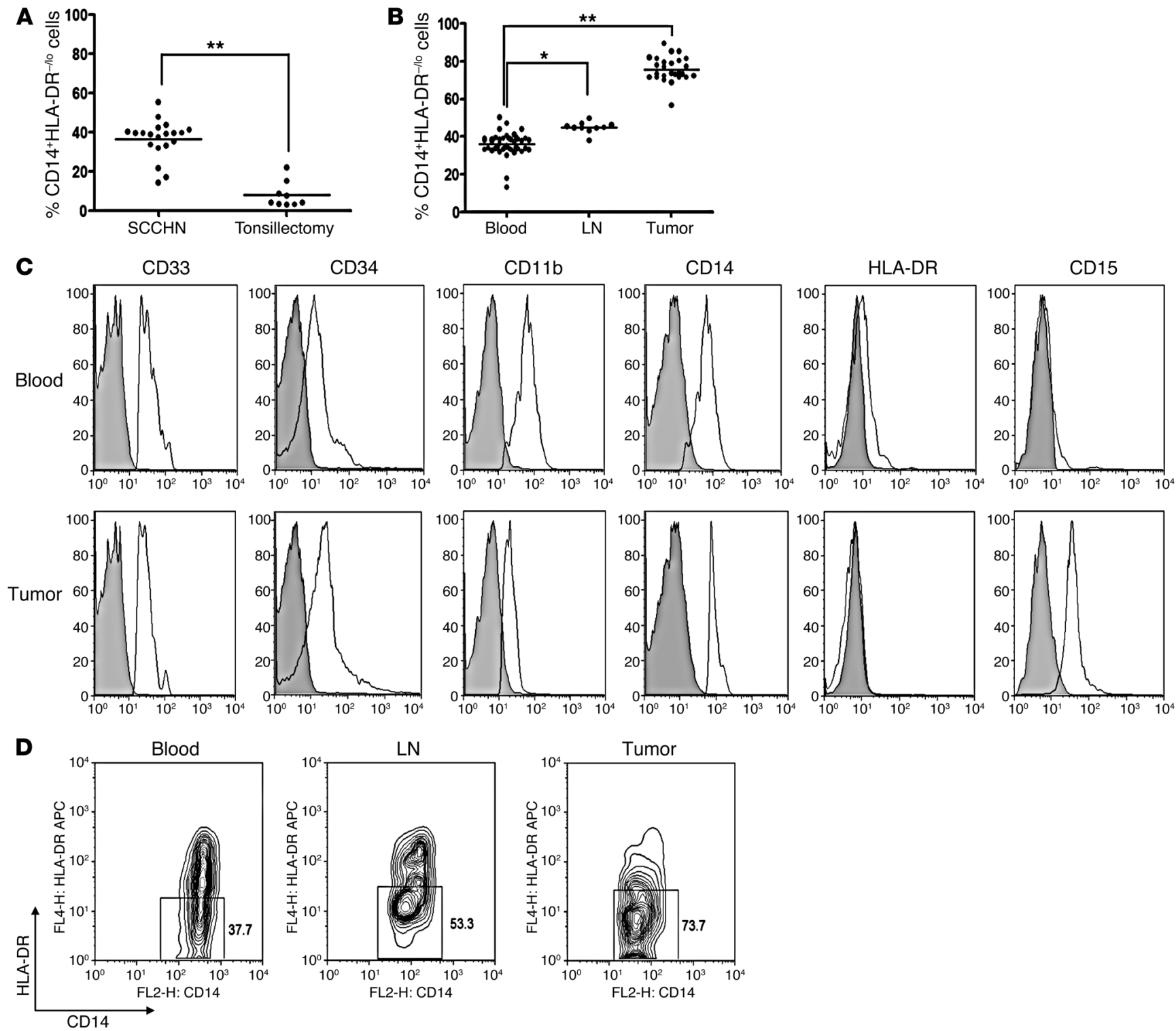

Figure 1

CD14+HLA-DR ${ }^{-/ 10}$ distribution and phenotypes in the blood, LNs, and tumors from HNSCC patients. (A) Relative abundance of circulating CD14+HLA-DR-1lo MDSC (with respect to CD11 $\mathrm{b}^{+}$cells prior to sorting in a given compartment) on HNSCC patients $(\mathrm{SCCHN})(n=20)$ versus patients with chronic tonsillitis $\left(n=9 ;{ }^{* *} P<0.01\right)$. The mean CD11 $\mathrm{b}^{+}$cells from these 2 patient populations were not statistically different. (B) Relative abundance of CD14+HLA-DR ${ }^{-10} \mathrm{MDSC}$ from PB $(n=37)$, DLNs $(n=10)$, and the primary tumor from $\mathrm{HNSCC}$ patients $\left(n=27 ;{ }^{*} P<0.05\right.$, $\left.{ }^{\star \star} P<0.01\right)$. (C) Representative histograms of MDSC markers on CD14+HLA-DR-/lo cells from PB and tumor with isotype controls as shaded areas. The expression of CD33, CD34, CD14, and CD11b was similar between blood and tumor-infiltrating MDSC. There was a differential expression of CD15 in this population between PB and the primary tumor. (D) Circulating CD14+HLA-DR-/lo cells demonstrated skewing toward higher percentage of the HLA-DR int population, while the tumor and LNs had a greater percentage of the HLA-DR- phenotype (central and right panels, respectively). Numbers represent the percentages of cells in the indicated boxes.

and MDSC in human tumor tissue has not been well characterized. Recently, murine MDSC from the periphery was found to differentiate into tumor-associated macrophages (TAM) in the tumor tissue in an HIF1 $\alpha$-dependent manner, but such studies have not been explored extensively in the human system (32).

In this study, we were able to sort CD14+HLA-DR ${ }^{-/ 10}$ MDSC from HNSCC patients from the 3 different compartments (tumor, draining LNs [DLNs], and PB) to characterize their phenotype and their suppressive function and to evaluate the STAT3 signaling in each of the compartments as it relates to their suppressive function.

\section{Results}

CD14 $14^{+} H L A-D R^{-/ l o}$ cell distribution and phenotypic markers vary in the tumor tissue, DLNs, and PB from buman HNSCC patients. We examined the abundance of CD14+HLA-DR $-1 /$ cells in the PB of HNSCC patients undergoing surgical ablation and found that there was 
A

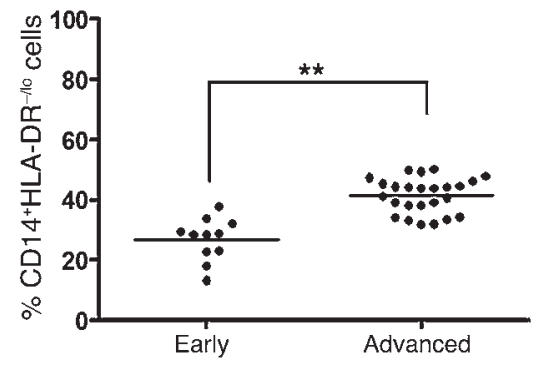

B
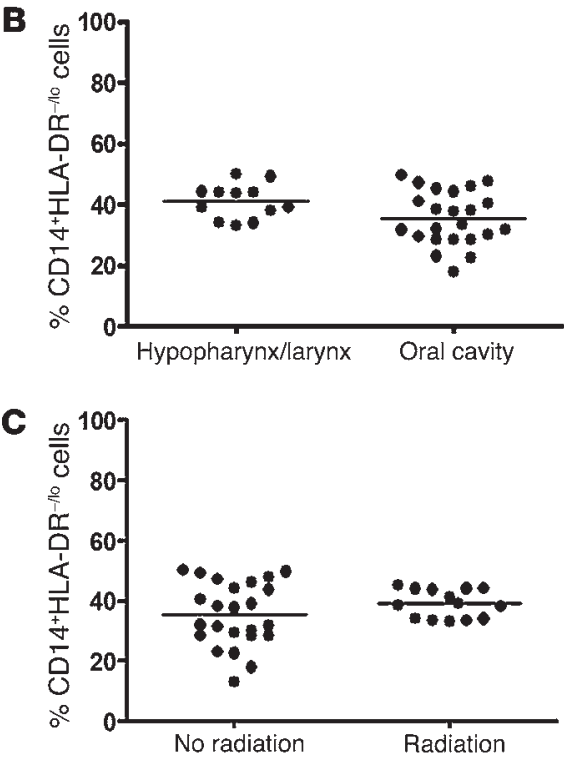

a larger accumulation of these potentially suppressive cells in HNSCC patients in comparison with patients suffering from chronic inflammatory disease. Mean CD14 ${ }^{+} \mathrm{HLA}^{-D R} \mathrm{P}^{-/ 10}$ cells relative to total CD11 $\mathrm{b}^{+}$myeloid cells in HNSCC patients in PB was $37.3 \% \pm 3.3 \%$ in comparison with $6.6 \% \pm 2.1 \%$ in patients with chronic tonsillitis (Figure 1A). When we analyzed CD14+HLA-DR-/1o cells in the tumor, the DLNs, and the PB at the time of surgical resection, we also noted differential distribution among these 3 sites. We found the highest percentage of CD $14^{+} \mathrm{HLA}-\mathrm{DR}^{-/ 1 \mathrm{lo}}$ cells (per CD $11 b^{+}$cells) in the tumor $(70.6 \% \pm 2.3 \%)$, followed by the DLNs with $44.4 \% \pm 2.1 \%$ and PB with $37.3 \% \pm 1.7 \%$ (Figure $1 B$ ).

Further phenotypic analysis of these cells for surface markers showed that these cells were consistent with MDSC. CD14 HLA$\mathrm{DR}^{-/ 10}$ cells expressed the myeloid markers CD11b, CD33, and CD34 (Figure 1C) as well as ARG1 and ROS (see below). In the tumor tissue, these cells also expressed CD15, while the matched cells from the PB did not. Furthermore, we noted that CD14 HLA$\mathrm{DR}^{-/ 1 \mathrm{lo}}$ cells displayed differential patterns of expression of MDSC markers among the tumor, DLNs, and PB. The most notable was the differential pattern of expression of HLA-DR and CD15 (Figure 1C). Circulating CD14+HLA-DR ${ }^{-/ 10}$ cells were skewed toward HLADR int distribution, while the tumor and LN CD14+HLA-DR-/lo cells predominantly displayed the HLA-DR- phenotype (Figure 1D). The number of patients with sufficient numbers of sorted CD14-HLA-DR-/lo cells was variable and was not included in the current clinical correlation analysis. Of the sorted CD14-HLA$\mathrm{DR}^{-/ 10}$ cells, we noted that these also suppressed autologous $\mathrm{T}$ cells (Supplemental Figure 1B; supplemental material available online

\section{Figure 2}

Abundance of CD14+HLA-DR-/lo MDSC-like cells correlated with staging but not head and neck subsites or previous radiation treatment. (A) Percentages of circulating MDSC among advanced stage (stage III and IV) HNSCC patients versus early stage (I and II) HNSCC patients ${ }^{* *} P<0.01$ ) with respect to CD11 $\mathrm{b}^{+}$. (B) Abundance pattern of MDSC in different subsites of HNSCC. (C) Percentages of circulating MDSC in HNSCC patients with or without radiotherapy exposure. The mean $C D 11 b^{+}$cells from these 2 patient populations were not statistically different.

with this article; doi:10.1172/JCI60083DS1). When we analyzed these cells for macrophage markers, we found that these cells did not express F4/80, CD68, and CD204 in the circulating or tumorinfiltrating MDSC (Supplemental Figure 2).

When we examined the morphologic features of peripheral and tumor infiltration cells, both peripheral and tumor-infiltrating CD14 ${ }^{+}$HLA-DR-/lo cells displayed predominantly mononuclear features (Supplemental Figure 3). As noted in Figure 1, the tumorinfiltrating CD14 ${ }^{+} \mathrm{HLA}-\mathrm{DR}-/ \mathrm{lo}$ cells also expressed CD15. Because of this heterogeneity in the histological phenotype categorization in CD14-CD $15^{+}$MDSC even after the sorting, we initially focused our attention toward the more homogeneous $\mathrm{CD} 14^{+}$monocytic population to examine critical signaling pathways that can be definitively linked to MDSC's suppressive function.

The abundance of $C D 14^{+} H L A-D R^{-/ l o}$ cells correlates with staging, but not subsites or previous radiation treatment. In melanoma patients, there was a positive correlation between stage and peripheral MDSC abundance (33). Likewise, we found that there was a significant increase in the percentage of circulating CD14 ${ }^{+} \mathrm{HLA}-\mathrm{DR}^{-/ \mathrm{lo}}$ cells among advanced stage (stage III and IV) HNSCC patients $(41.43 \pm 1.16 \%)$ as compared with early stage (stage I and II) HNSCC patients $(26.89 \% \pm 2.14 \%)$ (Figure $2 \mathrm{~A})$. There was no difference in the percentage of circulating CD14 $4^{+} \mathrm{HLA}-\mathrm{DR}-/ 1 \mathrm{lo}$ cells by tumor subsites in HNSCC (Figure 2B). We were unable to analyze any substantial population of oropharyngeal HNSCC sites due to the low volume of surgical candidates for these patients in our institution. Exposure to external beam radiotherapy did not appear to affect the percentage of circulating CD14 ${ }^{+} \mathrm{HLA}-\mathrm{DR} \mathrm{R}^{-/ 10}$ cells (Figure 2C). Comparable analysis restricted to laryngeal subsites showed similar results (data not shown).

CD14 $14^{+} H L A-D R^{-1 / 0}$ cells are MDSC that suppress autologous T cell proliferation - MDSC from the tumor and the DLNs have greater suppressive potential than MDSC from PB. CD14 HLA-DR-/lo cells above isolated from HNSCC patients were found to suppress autologous $\mathrm{T}$ cell proliferation in vitro (Figure 3A). At 2:1 T cell/MDSC titer, marked suppression was noted in the tumor, DLNs, and PB. Even at a 4:1 ratio, there was a tendency toward suppressive function. We initially used the $C D 11 b^{+} D R^{\text {hi }}$ myeloid population as our negative control for these suppression assays, but these $\mathrm{DR}{ }^{\mathrm{hi}}$ cells induced variable proliferative responses on $\mathrm{T}$ cells and never displayed suppressive functions (Supplemental Figure 4). Because of this problem with using nonimmunosuppressive myeloid cells, we presented raw ${ }^{3} \mathrm{H}$-thymidine uptake numbers in cpm rather than percentage of suppression. Notably, CD14 ${ }^{+} \mathrm{HLA}^{-\mathrm{DR}^{-} / \mathrm{lo} \mathrm{MDSC}}$ from the tumor and the DLNs have greater ability to suppress autologous $\mathrm{T}$ cells in comparison with matched PB MDSC (Figure $3 \mathrm{~A}$ ). This ability to suppress $\mathrm{T}$ cell proliferation correlated with the level of IFN- $\gamma$ detected in these assays (Supplemental Figure 5). As further confirmation that these were MDSC, 2 critical down- 
A

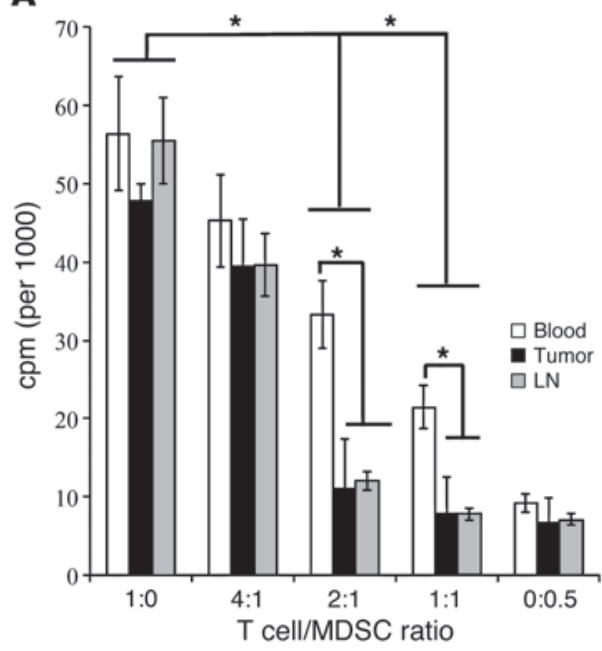

B
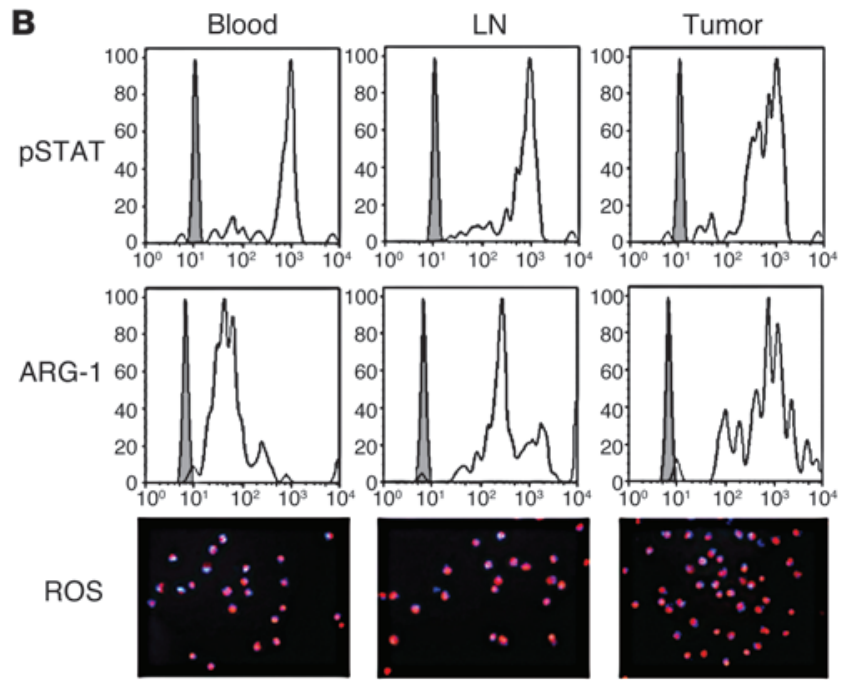
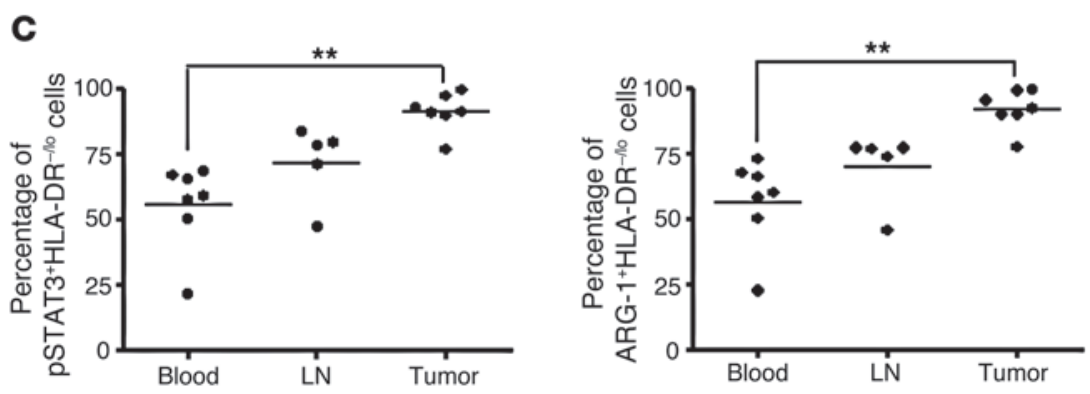

\section{Figure 3}

CD14+HLA-DR-1lo MDSC from blood, LNs, and tumor suppress autologous T cell proliferation and express high levels of pSTAT3, ARG1, and ROS. (A) Circulating CD14 ${ }^{+} \mathrm{HLA}^{-D R^{-/ l o}}$ cells suppress autologous $\mathrm{T}$ cell proliferation at a 2:1 $\mathrm{T}$ cell/MDSC ratio. Tumor and LN MDSC showed a greater level of suppressive activity, particularly at 2:1 and 1:1 ratios (T cell/MDSC) $\left({ }^{\star} P<0.05\right)$. (B) CD14+HLA-DR-1/0 MDSC from blood, LNs, and tumor express high levels of pSTAT3, ARG1, and ROS (DHE-stained cells). Original magnification, $\times 200$. (C) MDSC from PB, LNs, and tumor have differential expression of pSTAT3 and ARG1, with higher pSTAT3 and ARG1 expression levels noted in the tumor in comparison with PB. The percentages are calculated by relative abundance with respect to total CD14+HLA-DR-/lo cells $\left({ }^{* *} P<0.01\right)$.

stream mediators of suppressive function, ARG1 and ROS, were found to be present in these cells (Figure 3B).

Given some of these correlative data, we determined whether some of the common phenotypic markers would correlate with their suppressive activity. We sorted $\mathrm{DR}^{\mathrm{lo}}$ and $\mathrm{DR}^{-}$separately from all 3 sites to test whether HLA expression can further enrich MDSC suppressive activity in these 2 populations. The suppressive activity between $\mathrm{DR}^{\mathrm{lo}}$ and $\mathrm{DR}^{-}$MDSC did not differ in their suppressive activity in any given compartment, while CD14 ${ }^{+} \mathrm{HLA}-$ $\mathrm{DR}^{\text {int }}$ and $\mathrm{CD} 14^{+} \mathrm{HLA}-\mathrm{DR} \mathrm{R}^{\mathrm{lo}-}$ MDSC subsets from the tumor consistently had increased suppressive activity in comparison with their counterparts in the periphery (data not shown).

MDSC express PSTAT3, and this expression correlates with ARG1 expression. Given the functional significance of STAT3 signaling in myeloid cells in mice, we analyzed intracellular phosphorylated STAT3 (pSTAT3) and ARG1 expression level in the CD14+HLA$\mathrm{DR}^{-/ l o}$ MDSC from HNSCC patients. We observed high expression of both markers in the CD14+HLA-DR ${ }^{-/ 10}$ MDSC from tumor, DLNs, and PB (Figure 3B). Interestingly, MDSC from the 3 different sites had differential expression levels of pSTAT3 (Figure 3C). The tumor-infiltrating CD14+HLA-DR-/lo MDSC had statistically higher expression of PSTAT3 in comparison with the DLNs and
PB. Accordingly, we also detected a similar correlation in the level of expression of ARG1 in the 3 different sites.

Blockade and suppression of PSTAT3 ablates the suppressive activity of MDSC and decreases the expression and activity of ARG1. In our correlation analysis, the increased level of PSTAT3 in the tumor correlated with the greater suppressive activity of tumor-infiltrating MDSC in HNSCC patients. We therefore directly tested the significance of STAT3 signaling in MDSC in the human HNSCC by inhibiting STAT3 signaling using 2 independent methods. We used either Stattic, a specific small molecule inhibitor of PSTAT3, or siRNA suppression via lentiviral vector to inhibit STAT3 signaling. Both methods showed that inhibition of STAT3 signaling abrogated the suppressive function of $\mathrm{CD} 4^{+} \mathrm{HLA}^{-D R^{-/ 10}}$ MDSC from the tumor tissue as well as from the PB (Figure 4). The suppressive activity of circulating and tumor-infiltrating CD $14^{+} \mathrm{HLA}-$ $\mathrm{DR}^{-/ 10}$ cells decreased with Stattic at 2:1 and 1:1 ratios (Figure 4, A and B). Equivalent suppression was also obtained with the siRNA STAT3 suppression (Figure 4, C and D).

Interestingly, both forms of STAT3 signaling inhibition on CD14 ${ }^{+}$HLA-DR - lo $M D S C$, either with Stattic $(10 \mu \mathrm{M})$ or with the siRNA (Figure 5A), also affected the expression of ARG1 in these cells (Figure 5B). When we analyzed ARG1 activity in these MDSC 


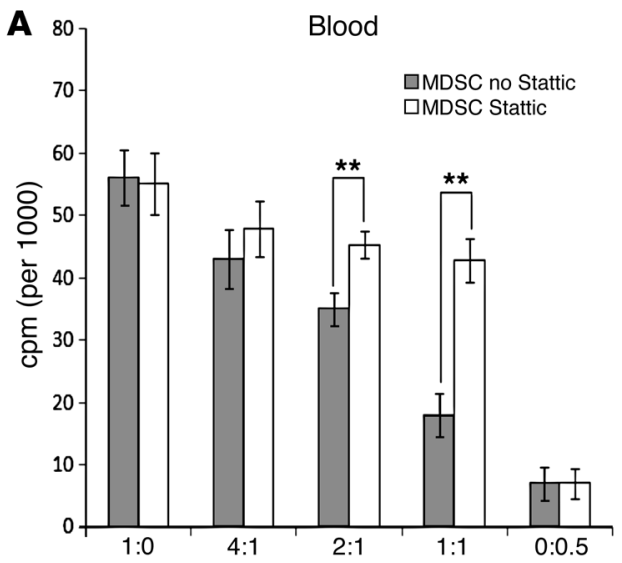

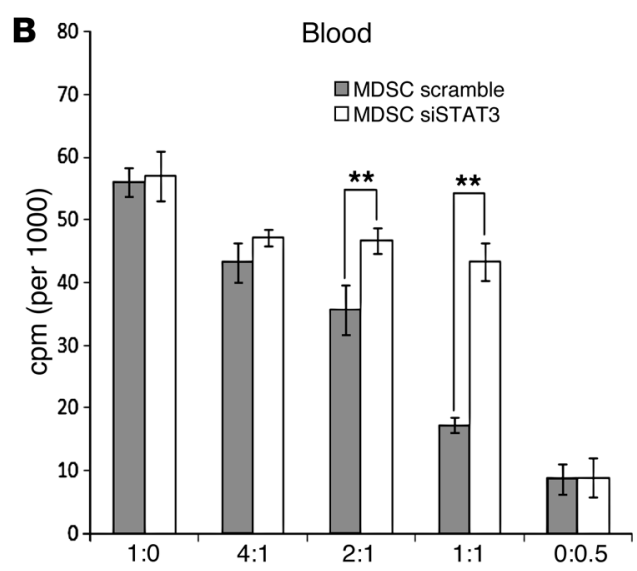

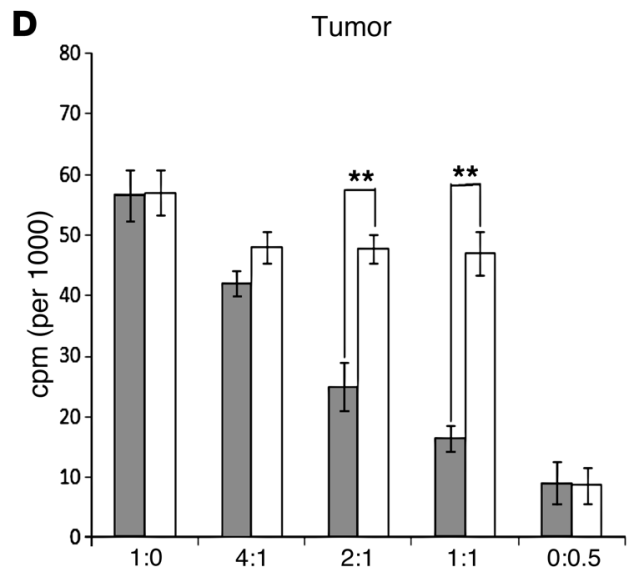

\section{Figure 4}

Inhibition of STAT3 signaling ablates the suppressive activity of CD14+HLA-DR-/lo MDSC from HNSCC patients. Both pSTAT3 small molecule inhibitor (Stattic at $10 \mu \mathrm{M})(\mathbf{A}$ and $\mathbf{C})$, and STAT3 siRNA (B and $\mathbf{D}$ ) were able to block the functional suppressive capability of blood ( $\mathbf{A}$ and $\mathbf{B})$ and tumor-infiltrating (C and $\mathbf{D})$ MDSC $\left({ }^{\star \star} P<0.01\right) . x$ axis shows the ratio of autologous $T$ cell/MDSC in the $T$ cell stimulation assay.

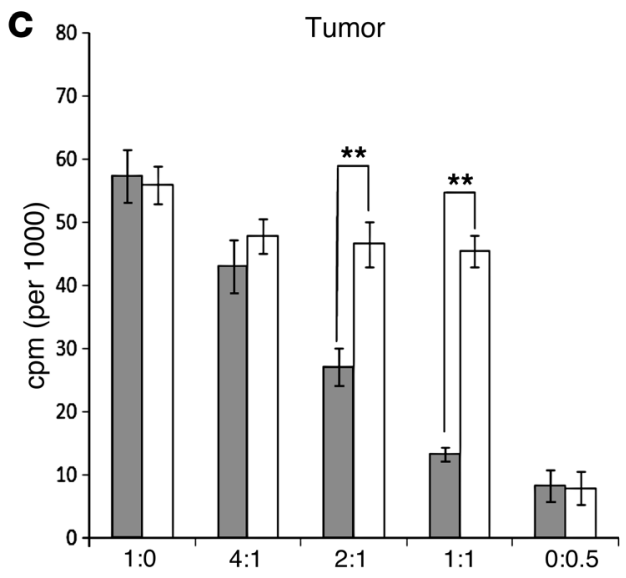

treated with Stattic, we found significant decreases in their enzymatic activity, both in the PB and in the tumor tissue (Figure 5C). These data suggest that STAT3 may regulate ARG1 in MDSC.

pSTAT3 can bind to buman ARG1 promoter elements. Given this functional correlation between PSTAT3 and ARG1 expression, we hypothesized that PSTAT3 can regulate ARG1 at the transcriptional level. We first analyzed the promoter elements of human ARG1 to screen for STAT3-binding elements. Using Vista genomic tools, we found 13 potential pSTAT3-binding sites in the ARG1 promoter (34). Using ChIP assay on sorted CD14 ${ }^{+}$HLA$\mathrm{DR}^{-/ 10}$ MDSC from $\mathrm{PB}$, we found that PSTAT3 is bound to multiple binding elements in the ARG1 promoter regions (Figure 6 and Supplemental Table 2).

The suppressive function of MDSC is rescued with the addition of ARG1 in pSTAT3-blocked MDSC. Since PSTAT3 blockade in CD14 ${ }^{+}$HLA$\mathrm{DR}^{-/ 10}$ MDSC decreased the expression and the activity of ARG1, we determined whether we could rescue the suppressive function of STAT3 with ARG1 repletion. Previous reports demonstrated that MDSC secrete ARG1, possibly via exocytosis, rather than indirectly deplete L-arg intracellularly $(35,36)$. We directly tested the supernatants harvested from cultured MDSC to ensure ARG1 activity was present in the supernatant, thereby validating our rescue method (Supplemental Figure 6). We had previously titrated the ARG1 level in these human MDSC (Figure 5) and found that the physiological concentrations ranged from 10 to $50 \mathrm{nM}$. The addition of physiological concentrations of human recombinant ARG1 to MDSC treated with Stattic effectively rescued the suppressive activity of the CD14+HLA-DR-/lo MDSC $(P<0.01)$ (Fig- ure $7 \mathrm{~A})$. There appeared to be a trend toward dose dependence for ARG1, but this was not statistically significant. The addition of ARG1 without pSTAT3 blockade did not alter the suppressive activity of the MDSC.

Repletion with L-arg or treatment with nor-NOHA, the ARG1 inhibitor, partially abrogated MDSC's suppressive function as observed previously (Figure 7B). The addition of Stattic in combination with either L-arg or nor-NOHA brought the abrogation of suppressive function as seen with Stattic treatment alone, which is further consistent with the hypothesis that STAT3 is upstream of ARG1. In other words, the lack of additive affect with Stattic and nor-NOHA implies that these 2 inhibitors are affecting the same pathway. Finally, blockade of PSTAT3 had the greatest abrogation of suppressive function in comparison with L-arg repletion or nor-NOHA treatment $(P<0.01)$ (Figure 7B). These data further corroborate our hypothesis, but also suggested that STAT3 may regulate other downstream mediators of lymphocyte suppression.

\section{Discussion}

MDSC are primarily defined by their suppressive function (37), and we have demonstrated that CD $14^{+} \mathrm{HLA}^{-D R^{-/ 10}}$ MDSC from HNSCC patients can be sorted from the primary tumor, the DLNs, and the PB to study critical signaling pathways as it relates to their suppressive function. One critical pathway in the tumor and periphery is that mediated by STAT3 signaling in the monocytic MDSC population. In multiple murine models, STAT3 signaling in the hematopoietic myeloid cells induced a tumor-promoting effect in vivo. Consistent with recent reports in cancer patients 

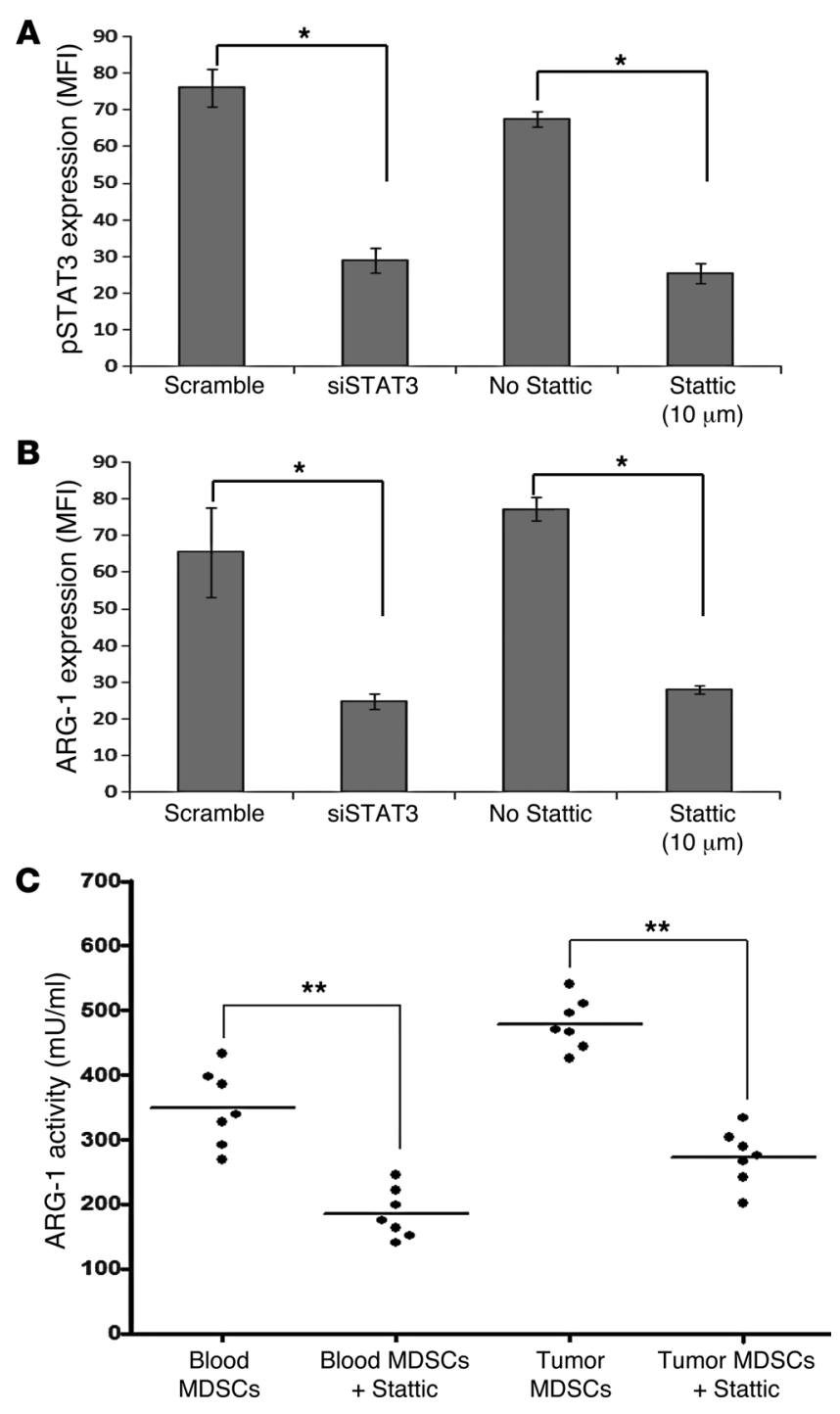

noted above, we also demonstrated that STAT3 signaling in MDSC from HNSCC patients has a functional role in its ability to suppress autologous $\mathrm{T}$ cell proliferation in both the tumor and the PB. STAT3 inhibition decreased the level of ARG1 and the suppressive activity in MDSC. More importantly, we also found that pSTAT3 can bind to the ARG1 promoter, and using biochemical methods, we demonstrated that ARG1, a key downstream modulator of human MDSC function, is regulated by PSTAT3.

We broadly sorted CD $14^{+} \mathrm{HLA}_{-} \mathrm{DR}^{-/ 10}$ myeloid cells from the tumor, DLNs, and PB of HNSCC patients. The number of patients with sufficient numbers of sorted CD14- cells was limited, and these cells were not included in the current analysis. Of

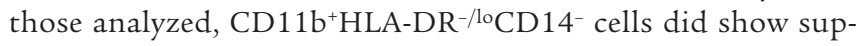
pressive function (Supplemental Figure 1). In the murine models, MDSC subsets were initially categorized as either granulocytic or mononuclear on the basis of Ly6C and Ly6G expression with differential suppressive functions (38). Comparable categorization of human MDSC into monocytic $\left(\mathrm{CD} 14^{+}\right.$) versus granulocytic $\left(\mathrm{CD} 14^{-} \mathrm{CD} 15^{+}\right)$has been proposed, but the specific surface markers of human MDSC subsets in cancer patients is still unclear (39). In our study, we initially sorted the monocytic CD $14^{+} \mathrm{DR}{ }^{10 /-}$

\section{Figure 5}

Inhibition of PSTAT3 decreases the expression and the activity of ARG1 on CD14+HLA-DR ${ }^{-1 / 0}$ MDSC. (A) Inhibition of STAT3 signaling on CD14+HLA-DR-1/0 MDSC by Stattic $(10 \mu \mathrm{M})$ or siSTAT3 appropriately decreased the level of pSTAT3 $\left({ }^{*} P<0.05\right)$. (B) Intracellular level of ARG1 is decreased with 2 independent methods of STAT3 signaling inhibition. $y$ axis shows MFI $\left({ }^{\star} P<0.05\right)$. (C) ARG1 activity of circulating and tumor-infiltrating CD14+HLA-DR ${ }^{-/ 10}$ MDSC after pSTAT3 inhibition with Stattic $\left.{ }^{* *} P<0.01\right)$.

population, but in the tumor-infiltrating MDSC, our sorted CD14+ cells also expressed CD15 antigen. These data are consistent with data from Mandruzzato et al. (13) that showed that CD14 and CD15 expression overlap in MDSC from colon cancer and melanoma patients. In other words, these data suggest that CD14 and CD15 may not clearly delineate the morphologic subtypes of MDSC in the human system. Our findings corroborate the phenotypic heterogeneity found in tumor-infiltrating MDSC sorted from melanoma patients (40).

To directly test the ability of CD15 and CD14 markers to separate the MDSC populations into polymorphonuclear and monocytic cells, we separately sorted myeloid CD $14^{\text {hi HLA-DR }}{ }^{\text {lo }}$ as well as $\mathrm{CD} 14^{\text {lo }} \mathrm{CD} 15^{\text {hi }} \mathrm{HLA}-\mathrm{DR}{ }^{\text {lo }}$ cells from both the tumor and the blood from HNSCC after Ficoll density gradient. The CD14hiHLA$\mathrm{DR}^{\mathrm{lo}}$ population was found to be $\mathrm{CD} 15^{-}$and was predominantly monocytic, while the CD $14^{\text {lo }} \mathrm{CD} 15^{\text {hi }} \mathrm{HLA}-\mathrm{DR}{ }^{\mathrm{lo}}$ population had on average equal distribution of monocytic and polymorphonuclear cells (Supplemental Figure 4). These data further underscored the limitation of using the CD15 antigen to delineate PMN MDSC subpopulations from cancer patients. We are currently focused on the ability to isolate a more homogenous PMN MDSC from the $\mathrm{PB}$ and the tumors of HNSCC patients from matched samples.

Recent studies of tumor-infiltrating MDSC from melanoma patients showed minimal suppressive function (40). However, they also reported that their tumor-infiltrating immature myeloid cells had higher levels of HLA-DR, suggestive of TAM or more differentiated myeloid cells that may be the source of their inability to suppress $\mathrm{T}$ cells. Other labs, as ours, demonstrated suppressive tumor-infiltrating MDSC in HNSCC specimens (32, 41). These conflicting results, however, attest to the importance of examining the tumor-infiltrating MDSC population $(42,43)$. In tumor-bearing mice, $\mathrm{CD} 11 \mathrm{~b}^{+} \mathrm{Gr}-1^{+} \mathrm{MDSC}$ from the tumor and the peripheral lymphoid organs have profound functional differences in their ability to mediate differential $\mathrm{T}$ cell-suppressive responses. While tumor-infiltrating MDSC suppressed both antigen-specific and non-antigen-specific $T$ cell function, the peripheral MDSC only suppressed antigen-specific T cell function. In the tumor microenvironment, murine MDSC also differentiates into HIF1 $\alpha$-dependent TAM. In our human system, there was a differential suppressive function between the tumorinfiltrating and PB MDSC as well (Figure 3A). In our preparation, we sorted specifically for low HLA-DR expression. Testing to see whether our sorted MDSC suppress tumor antigen-specific T cells was not feasible at this point in time, but we did examine the tumor-infiltrating MDSC for macrophage markers, and we did not see expression of human F4/80, CD68, and CD204 on these populations (Supplemental Figure 2). The apparent differences in these findings may stem from the fact we had enriched for nonmacrophage population in our sorting methods, and these results do not exclude the possibility of immature myeloid 


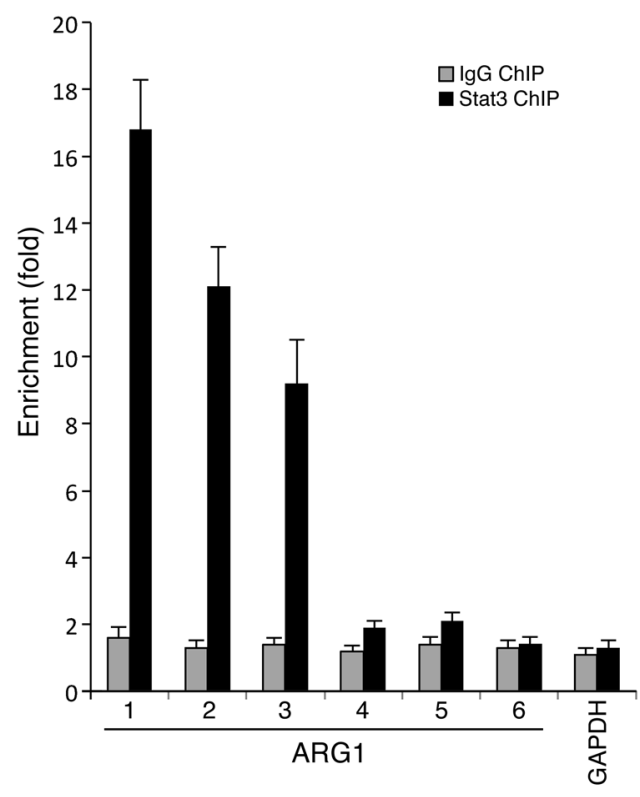

Figure 6

STAT3 binds to the promoter region of ARG1 of MDSC. ChIP assay demonstrated pSTAT3 binding to ARG1 promoter regions at 3 of the 6 potential binding sites (from a total of 12 sites matching the consensus sequences generated by Vista genomic program). The sequence of the human ARG1 promoter region with the 6 potential pSTAT3-binding sites is shown in Supplemental Table 2.

cells with the potential for differentiation into macrophages. With our greater homogeneity in our purification scheme, we may have isolated a TAM precursor MDSC subpopulation that was found to possess enhanced suppressive function in comparison with the peripheral MDSC. Another possibility is that the $\mathrm{CD} 14^{+} \mathrm{CD} 15^{+} \mathrm{DR}^{-}$population may be another subset of MDSC that is correlated with enhanced suppressive function.

With the ability to study matched MDSC from the tumor and the periphery, we sought to examine regulatory factors in MDSC that could control its suppressive function. In murine models, pSTAT3 had been shown to regulate the expansion of myeloid progenitors as well as MDSC $(21,44)$. However, unlike STAT1 and STAT6, STAT3 has not been reported to directly regulate the MDSC's suppressive function (1). In TAM in mice, experiments with STAT1 and STAT6 knockout mice initially suggested that ARG1 activity may be primarily dependent on STAT1 (26). More recent reports in murine alternatively activated macrophages demonstrated that STAT3 can regulate ARG1 $(22,29)$. Moreover, human MDSC's immunosuppressive activity from cancer patients was found to be STAT3 dependent $(18,19)$.

Given this controversy over the relationship between STAT3 and MDSC's suppressive function, we evaluated the relationship between PSTAT3 and the T cell-suppressive function in human MDSC from HNSCC patients. We analyzed the promoter sequences of ARG1 for potential STAT3-binding elements and found that there were 13 potential STAT3-binding elements. CHiP assay demonstrated that PSTAT3 proteins are bound to 3 of these binding elements (Figure 6). We also demonstrated that both siRNA suppression and PSTAT3 inhibition using a STAT3-specific inhibitor could abrogate human MDSC's suppressive function as well as decrease the level and activity of ARG1 (Figure 5). When we replen- ished the physiological level of ARG1 in pSTAT3-blocked MDSC, we were able to rescue MDSC's suppressive function (Figure 7). Although the mechanism of ARG1 secretion in MDSC is controversial, we confirmed that these cells can secrete ARG1, as shown in Supplemental Figure 6, validating our rescue experiments. Analysis with nor-NOHA and L-arg replenishment partially replicated the effect of PSTAT3 signaling inhibition in the MDSC. Cumulatively, these results demonstrate that STAT3 signaling is upstream of the activity of ARG1 that mediates the suppressive function of $T$ cell proliferation in HNSCC patients.

With the recent reports of several STAT3-dependent genes that have been reported to play critical roles in MDSC's function, there may be multiple pathways of STAT3-dependent immunosuppression. While we demonstrated a direct regulation of ARG1, Bronte and others have shown that STAT3-dependent C/EBP $\beta$ transcription factor is critical in regulating immunosuppression (20). Others have shown that HIF1 $\alpha$, another STAT3-dependent gene, mediates the differentiation into tumor-infiltrating macrophages (32). With our ability to sort sufficient numbers of matched blood and tumor-infiltrating MDSC from HNSCC patients, we can potentially test some of these STAT3 dependent pathways in MDSC.

We are currently studying other downstream STAT3-dependent genes in both tumor-infiltrating and peripheral MDSC from HNSCC patients. Of particular interest is the differential expression of other STAT3-dependent genes between the tumor and the periphery. Although only STAT1-null mice failed to upregulate ARG1 and iNOS, promoters of both of these downstream mediators of MDSC function have STAT3-binding elements in the human system (data not shown). This is particularly relevant for MDSC, since there are now several anticarcinogenic STAT3-inhibiting molecules that are in clinical trials, so their secondary effects on human MDSC function require further investigation $(45,46)$.

The implication of STAT3's importance in MDSC function is clinically significant. For HNSCC patients, EGFR inhibition has demonstrated clinical efficacy, but its mechanism of action is still unclear $(47,48)$. EGFR is an upstream mediator of the JAK/ STAT3 pathway, so systemic EGFR inhibition may affect pSTAT3dependent MDSC's suppressive function (49). STAT3 signaling in MDSC may also be potentially modulated by IL-6 $(20,50)$, and IL-6 has been repeatedly found to be a critical biomarker of clinical prognosis in HNSCC (51). Interestingly, an independent lab recently demonstrated that curcumin, an herbal immunomodulatory molecule that have been previously found to suppress JAK2/ STAT3 signaling, demonstrated decreased tumor growth in vivo that correlated with IL-6-dependent downregulation of MDSC (52). Therefore, the mechanistic relationship between pSTAT3 and IL-6 in human MDSC may warrant further investigation now that we have demonstrated the functional significance of STAT3 signaling in MDSC from cancer patients.

\section{Methods}

MDSC isolation and flow cytometry analysis. MDSC characterized as CD14 HLA-DR-/lo were sorted from freshly obtained PB, DLNs, and/or tumor specimens from HNSCC patients undergoing surgical treatments at Johns Hopkins Hospital and Johns Hopkins Bayview Hospital. In some cases, $\mathrm{CD}^{1} 4^{-} \mathrm{CD} 15^{+} \mathrm{HLA}_{-} \mathrm{DR}^{-/ 10}$ cells were sorted. From PB, leukocytes were separated by a Ficoll gradient prior to sorting. From DLNs, the mechanically dissociated leukocytes were sorted after separating from debris with a $100-\mu \mathrm{m}$ cell strainer. For the tumor specimen, $1 \mathrm{~cm}$ tumor tissue was cut into small pieces and treated with Liberase (Roche) for 20 minutes at 

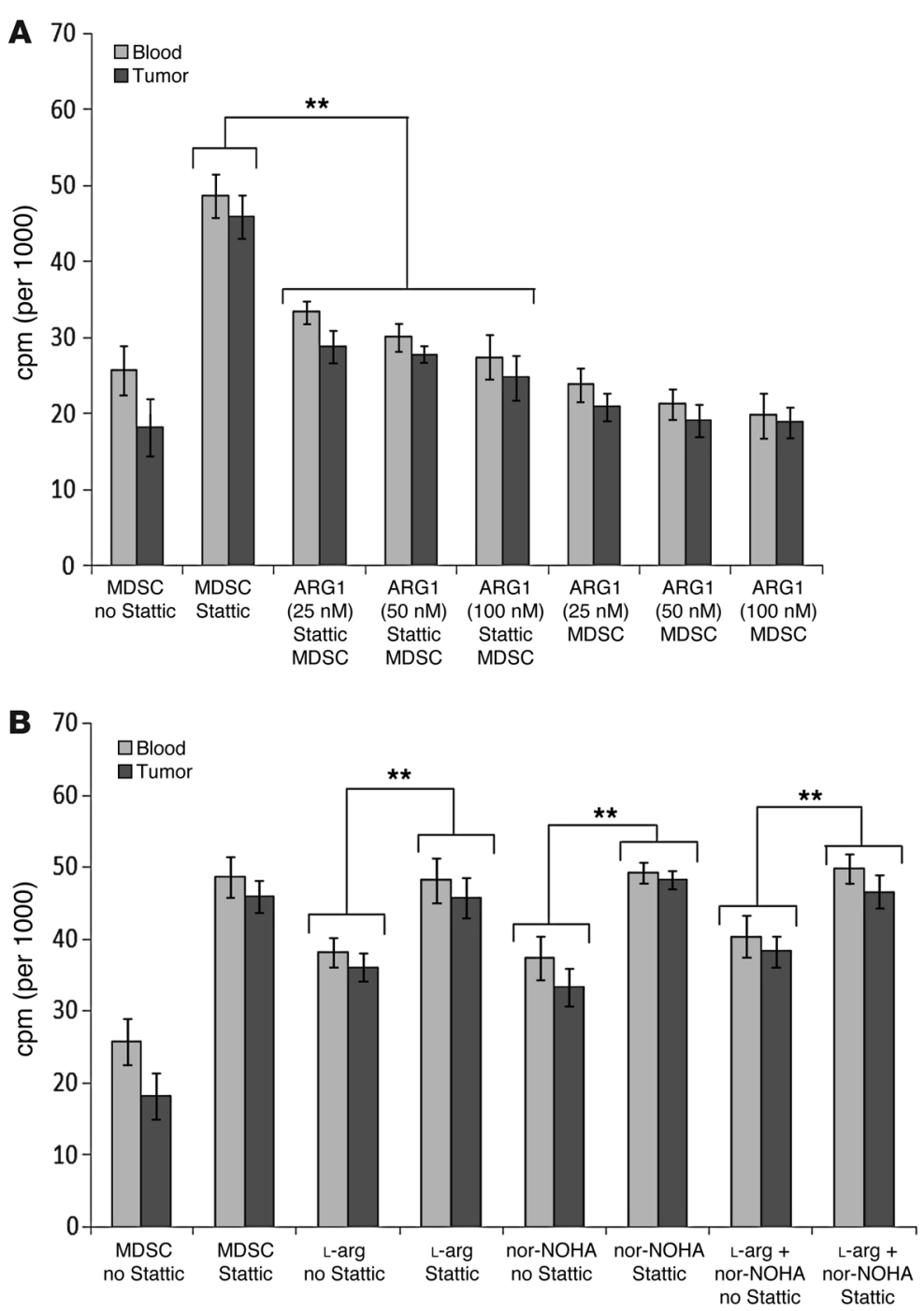

\section{Figure 7}

Suppressive function of MDSC can be rescued by adding back ARG1 to STAT3-blocked MDSC. (A) Addition of varying concentrations of human recombinant ARG1 to MDSC treated with Stattic rescued the suppressive function of the CD14 ${ }^{+}$HLA-DR ${ }^{-/ l o}$ MDSC $\left({ }^{* *} P<0.01\right)$. Addition of ARG1 without STAT3 inhibition did not affect the suppressive function of intact MDSC. T cell/MDSC ratio was 2:1. (B) Both L-arg and the ARG1 inhibitor nor-NOHA were also able to blunt the suppressive activity of MDSC, but STAT3 inhibition has a greater ablation of MDSC's suppressive function $\left({ }^{\star \star} P<0.01\right)$. Stattic and nor-NOHA does not have an additive effect on the ablation of MDSC suppressive function. All samples had MDSC, and the ratio of T cell/MDSC was 2:1.

sion assays were performed with 3\% FCS in RPMI 1640. In some instances, conditioned medium from cultured MDSC were used for this assay.

ROS staining. Superoxide production was measured by dihydroethidium (DHE) staining. Briefly, $5 \times 10^{5} \mathrm{CD} 14^{+} \mathrm{HLA}^{-D R^{-/ l o}}$ cells were incubated at $37^{\circ} \mathrm{C}$ in serum-free DMEM containing $2 \mu \mathrm{M}$ DHE for 1 hour to measure superoxide production. Stained cells were washed twice with PBS and analyzed with fluorescent microscopy (Johns Hopkins Hospital Imaging Core Facility).

STAT3 inhibition. Stattic, a STAT3-specific small molecule inhibitor (Calbiochem) and siRNA against STAT3 (siSTAT3) were used to inhibit STAT3 signaling. Stattic was diluted to $1 \%$ in DMSO (53). CD14 $4^{+} \mathrm{HLA}-\mathrm{DR}^{-/ 10}$ cells were treated with a concentration of $10 \mu \mathrm{M}$ Stattic at $37^{\circ} \mathrm{C}$ for 24 hours. Scrambled and STAT3 siRNA $(15 \mathrm{nM})$ were transduced into CD14+HLA$\mathrm{DR}^{-/ 1 \mathrm{lo}}$ cells using lentiviral vectors $(53,54)$.

ARG1 enzymatic assay. ARG1 activity was measured in circulating and tumor-infiltrating CD $14^{+} H L A-D R^{-/ l o}$ cell lysates with slight modifications, as previously described (55). In some instances, conditioned media from cultured MDSC were used for this assay. Briefly, cells were lysed with $100 \mu \mathrm{l}$ of $0.1 \%$ Triton X-100. After 20 minutes, $100 \mu \mathrm{l}$ of $25 \mathrm{mM}$ Tris-HCL was added. Then, $10 \mu \mathrm{l}$ of $10 \mathrm{mM} \mathrm{MnCL}_{2}$ were added to $100 \mu \mathrm{l}$

$37^{\circ} \mathrm{C}$. Suspension cells were separated from debris with a $100-\mu \mathrm{m}$ strainer and resuspended prior to sorting. Cells were sorted with MoFlo MLS sorter (Beckman Coulter) or FACSAria II cell sorter (BD) at Johns Hopkins Hospital Cell Cytometry Core Facility. Multicolor cell analysis was performed using the following antibodies: $\alpha \mathrm{CD} 14, \alpha \mathrm{CD} 4, \alpha \mathrm{CD} 11 \mathrm{~b}, \alpha \mathrm{CD} 33$, $\alpha \mathrm{CD} 34, \alpha \mathrm{CD} 15, \alpha H L A-D R$ (eBioscience), and $\alpha$ ARG1 (R\&D Systems). Cell-surface and intracellular staining were performed as indicated in the manufacturer's data sheet. For pStat3 (pY705) staining, cells were fixed in $2 \%$ paraformaldehyde/PBS for 10 minutes at $37^{\circ} \mathrm{C}$ and permeabilized by resuspending with vigorous vortexing in $500 \mu \mathrm{l}$ ice-cold $90 \%$ methanol per $10^{6}$ cells and incubated at $4^{\circ} \mathrm{C}$ for 30 minutes on ice. After PBS washing and resuspension, cells were stained with Alexa Fluor 647-conjugated pSTAT3 antibody (BD Biosciences) for 1 hour at room temperature. FlowJo software (TreeStar Inc.) was used for analysis. Isotype-matched antibodies were used as controls. Relative abundance of MDSC was measured with respect to total $\mathrm{CD} 11 \mathrm{~b}^{+}$cells in the sample $\left(\mathrm{CD} 14^{+} \mathrm{HLA}-\mathrm{DR}-/{ }^{-10} \mathrm{CD} 11 \mathrm{~b}^{+}\right)$.

$T$ cell suppression. Autologous $\mathrm{CD}^{+} \mathrm{T}$ cells were isolated during the sorting of MDSC in parallel and activated using $\alpha \mathrm{CD} 3$ and $\alpha \mathrm{CD} 28$ (eBioscience) absorbed onto plates. $\mathrm{T}$ cell proliferation and activation were measured after 72 hours using ${ }^{3} \mathrm{H}$-thymidine incorporation and IFN- $\gamma$ ELISA, respectively. MDSC or HLA-DR ${ }^{+}$myeloid controls were cocultured with autologous $\mathrm{CD}^{+} \mathrm{T}$ cells at different ( $\mathrm{T}$ cell/MDSC) ratios. $\mathrm{T}$ cell suppres- of lysate followed by 10 minutes of heating at $56^{\circ} \mathrm{C}$. The lysate was then incubated with $100 \mu \mathrm{l}$ of $\mathrm{L}-\arg \left(\mathrm{pH} 9.7\right.$ ) at $37^{\circ} \mathrm{C}$ for $30-60$ minutes. The reaction was stopped with $900 \mu \mathrm{l}$ of $\mathrm{H}_{2} \mathrm{SO}_{4} / \mathrm{H}_{3} \mathrm{PO}_{4} / \mathrm{H}_{2} \mathrm{O}(1 / 3 / 7, \mathrm{v} / \mathrm{v} / \mathrm{v})$. The urea concentration was measured at $540 \mathrm{~nm}$ after adding $40 \mu \mathrm{l}$ of $\alpha$-isonitrosopropiophenone (in $100 \%$ ethanol) followed by heating at $95^{\circ} \mathrm{C}$ for 30 minutes. One unit of enzyme is defined as the amount of enzyme that catalyzed the formation of $1 \mu \mathrm{mol}$ of urea per minute.

ARG1 supplementation and blockade. CD14+ ${ }^{+} \mathrm{HLA}-\mathrm{DR} \mathrm{R}^{-/ 10}$ cells treated with Stattic were incubated with L-Arg (Sigma-Aldrich) at $100 \mu \mathrm{M}$ and/or norNOHA (Cayman Chemical) at $20 \mu \mathrm{M}$ and then assayed for suppressor activity as described above. For ARG1 rescue assay, CD14+HLA-DR-/lo cells untreated or treated with Stattic were incubated with recombinant human ARG1 (R\&D systems) at varying doses (25-100 nM) overnight and then assayed for suppressor activity as described.

ChIP. ChIP assays were performed using the ChIP assay kit (Upstate) (54). Sorted MDSC cells were crosslinked and sonicated, and DNA was immunoprecipitated with anti-Stat 3 or isotype-matched control IgG (Cell Signaling) from the sonicated cell lysates and quantified using SYBR Green Real-time PCR analysis (Applied Biosystems). The primers used for ChIP assay are listed in Supplemental Table 1.

HङE staining of sorted CD14+HLA-DR-/lo MDSC cells. Sorted cells were placed on slides with Cytospin and fixed with $10 \%$ neutral buffered for- 
malin for 1 day. H\&E (Thermo Scientific) staining was performed by the Johns Hopkins Hospital Histology Core. Slides were analyzed with Nikon Eclipse F800 microscope.

Statistics. The differences in means and correlation analysis were evaluated with parametric (2-tailed Student's or paired $t$ test and Pearson's test) or nonparametric (Mann-Whitney $U$ or Wilcoxon's and Spearman's $P$ test) tests. Null hypothesis was rejected when $P$ value was less than 0.05 or 0.01 . Error bars throughout the figures represent SD of the mean.

Study approval. Our study was initiated after obtaining Johns Hopkins Hospital Institutional Review Board approval (NA_00019201 and NA_00012214), and the experiments were performed in compliance with these protocols. All subjects provided informed consent prior to participation, and the specimens were deidentified prior to analysis.

\section{Acknowledgments}

This work was supported by NIH K23-DE018464, a Flight Attendant Medical Research Institute grant, and an American College of Surgeons/Triological Career Developmental Grant (to Y.J. Kim).

Received for publication November 14, 2011, and accepted in revised form January 4, 2013.

Address correspondence to: Young Kim, Johns Hopkins Sidney Kimmel Comprehensive Cancer Center, Otolaryngology Head and Neck Surgery, 1650 Orleans St., CRB1, Room 4M61, Baltimore, Maryland 21231, USA. Phone: 410.550.0460; Fax: 410.550.5961; E-mail: ykim76@jhmi.edu.
1. Gabrilovich DI, Nagaraj S. Myeloid-derived suppressor cells as regulators of the immune system. Nat Rev Immunol. 2009;9(3):162-174.

2. Filipazzi $P$, et al. Identification of a new subset of myeloid suppressor cells in peripheral blood of melanoma patients with modulation by a granulocytemacrophage colony-stimulation factor-based antitumor vaccine. J Clin Oncol. 2007;25(18):2546-2553.

3. Hoechst B, et al. Myeloid derived suppressor cells inhibit natural killer cells in patients with hepatocellular carcinoma via the NKp30 receptor. Hepatology. 2009;50(3):799-807.

4. Pak AS, Wright MA, Matthews JP, Collins SL, Petruzzelli GJ, Young MR. Mechanisms of immune suppression in patients with head and neck cancer: presence of CD34(+) cells which suppress immune functions within cancers that secrete granulocytemacrophage colony-stimulating factor. Clin Cancer Res. 1995;1(1):95-103.

5. Gabitass RF, Annels NE, Stocken DD, Pandha HA, Middleton GW. Elevated myeloid-derived suppressor cells in pancreatic, esophageal and gastric cancer are an independent prognostic factor and are associated with significant elevation of the Th2 cytokine interleukin-13. Cancer Immunol Immunother. 2011;60(10):1419-1430.

6 . Solito S, et al. A human promyelocytic-like population is responsible for the immune suppression mediated by myeloid-derived suppressor cells. Blood. 2011;118(8):2254-2265.

7. Liu CY, et al. Population alterations of L-arginaseand inducible nitric oxide synthase-expressed

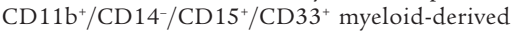
suppressor cells and $\mathrm{CD}^{+} \mathrm{T}$ lymphocytes in patients with advanced-stage non-small cell lung cancer. J Cancer Res Clin Oncol. 2010;136(1):35-45.

8. Serafini P, Borrello I, Bronte V. Myeloid suppressor cells in cancer: recruitment, phenotype, properties, and mechanisms of immune suppression. Semin Cancer Biol. 2006;16(1):53-65.

9. Greten TF, Manns MP, Korangy F. Myeloid derived suppressor cells in human diseases. Int Immunopharmacol. 2011;11(7):802-807.

10. Movahedi K, et al. Identification of discrete tumorinduced myeloid-derived suppressor cell subpopulations with distinct $\mathrm{T}$ cell-suppressive activity. Blood. 2008;111(8):4233-4244.

11. Filipazzi $P$, et al. Identification of a new subset of myeloid suppressor cells in peripheral blood of melanoma patients with modulation by a granulocytemacrophage colony-stimulation factor-based antitumor vaccine. J Clin Oncol. 2007;25(18):2546-2553.

12. Rodriguez PC, et al. Arginase I-producing myeloidderived suppressor cells in renal cell carcinoma are a subpopulation of activated granulocytes. Cancer Res. 2009;69(4):1553-1560.

13. Mandruzzato S, et al. IL4Ralpha ${ }^{+}$myeloid-derived suppressor cell expansion in cancer patients. J Immunol. 2009;182(10):6562-6568.

14. Rodriguez PC, Ochoa AC. Arginine regulation by myeloid derived suppressor cells and tolerance in cancer: mechanisms and therapeutic perspectives. Immunol Rev. 2008;222:180-191.

15. Talmadge JE. Pathways mediating the expansion and immunosuppressive activity of myeloid-derived suppressor cells and their relevance to cancer therapy. Clin Cancer Res. 2007;13(18 pt 1):5243-5248.

16. Ostrand-Rosenberg S, Sinha P. Myeloid-derived suppressor cells: linking inflammation and cancer. J Immunol. 2009;182(8):4499-4506.

17. Marigo I, Dolcetti L, Serafini P, Zanovello P, Bronte V. Tumor-induced tolerance and immune suppression by myeloid derived suppressor cells. Immunol Rev. 2008;222:162-179.

18. Poschke I, Mougiakakos D, Hansson J, Masucci GV, Kiessling R. Immature immunosuppressive CD $14^{+}$HLA-DR-/low cells in melanoma patients are Stat $3^{\text {hi }}$ and overexpress CD80, CD83, and DC-sign. Cancer Res. 2010;70(11):4335-4345.

19. Chalmin F, et al. Membrane-associated Hsp72 from tumor-derived exosomes mediates STAT3dependent immunosuppressive function of mouse and human myeloid-derived suppressor cells. J Clin Invest. 2010;120(2):457-471.

20. Marigo I, et al. Tumor-induced tolerance and immune suppression depend on the C/EBPbeta transcription factor. Immunity. 2010;32(6):790-802.

21. Zhang H, Nguyen-Jackson H, Panopoulos AD, Li HS, Murray PJ, Watowich SS. STAT3 controls myeloid progenitor growth during emergency granulopoiesis. Blood. 2010;116(14):2462-2471.

22. Csóka $B$, et al. Adenosine promotes alternative macrophage activation via $\mathrm{A} 2 \mathrm{~A}$ and $\mathrm{A} 2 \mathrm{~B}$ receptors. FASEB J. 2012;26(1):376-386.

23. Kortylewski M, et al. Inhibiting Stat 3 signaling in the hematopoietic system elicits multicomponent antitumor immunity. Nat. Med. 2005;11(12):1314-1321.

24. Sinha P, Okoro C, Foell D, Freeze HH, Ostrand-Rosenberg S, Srikrishna G. Proinflammatory S100 proteins regulate the accumulation of myeloid-derived suppressor cells. J Immunol. 2008;181(7):4666-4675.

25 . Cheng $P$, et al. Inhibition of dendritic cell differentiation and accumulation of myeloid-derived suppressor cells in cancer is regulated by S100A9 protein. J Exp Med. 2008;205(10):2235-2249.

26. Kusmartsev S, Gabrilovich DI. STAT1 signaling regulates tumor-associated macrophage-mediated T cell deletion. J Immunol. 2005;174(8):4880-4891.

27. Terabe $M$, et al. Transforming growth factor-beta production and myeloid cells are an effector mechanism through which CD1d-restricted T cells block cytotoxic T lymphocyte-mediated tumor immunosurveillance: abrogation prevents tumor recurrence. J Exp Med. 2003;198(11):1741-1752.

28. Delano MJ, et al. MyD88-dependent expansion of an immature GR-1(+)CD11b(+) population induces $T$ cell suppression and Th2 polarization in sepsis. J Exp Med. 2007;204(6):1463-1474.

29. Qualls JE, et al. Arginine usage in mycobacteriainfected macrophages depends on autocrine-para- crine cytokine signaling. Sci Signal. 2010;3(135):ra62. 30. Bronte V, Zanovello P. Regulation of immune responses by L-arginine metabolism. Nat Rev Immunol. 2005;5(8):641-654.

31. Yu H, Pardoll D, Jove R. STATs in cancer inflammation and immunity: a leading role for STAT3. Nat Rev Cancer. 2009;9(11):798-809.

32. Corzo CA, et al. HIF-1alpha regulates function and differentiation of myeloid-derived suppressor cells in the tumor microenvironment. J Exp Med. 2010; 207(11):2439-2453.

33. Diaz-Montero CM, Salem ML, Nishimura MI, Garrett-Mayer E, Cole DJ, Montero AJ. Increased circulating myeloid-derived suppressor cells correlate with clinical cancer stage, metastatic tumor burden, and doxorubicin-cyclophosphamide chemotherapy. Cancer Immunol Immunother. 2009;58(1):49-59.

34. Frazer KA, Pachter L, Poliakov A, Rubin EM, Dubchak I. VISTA: computational tools for comparative genomics. Nucleic Acids Res. 2004;32(Web Server issue):W273-W279.

35. Zea AH, et al. Arginase-producing myeloid suppressor cells in renal cell carcinoma patients: a mechanism of tumor evasion. Cancer Res. 2005;65(8):3044-3048.

36. Rotondo R, et al. Exocytosis of azurophil and arginase 1-containing granules by activated polymorphonuclear neutrophils is required to inhibit T lymphocyte proliferation. J Leukoc Biol. 2011;89(5):721-727.

37. Gabrilovich DI, et al. The terminology issue for myeloid-derived suppressor cells. Cancer Res. 2007; 67(1):425.

38. Movahedi K, et al. Identification of discrete tumorinduced myeloid-derived suppressor cell subpopulations with distinct $\mathrm{T}$ cell-suppressive activity. Blood. 2008;111(8):4233-4244.

39. Peranzoni E, et al. Myeloid-derived suppressor cell heterogeneity and subset definition. Curr Opin Immunol. 2010;22(2):238-244.

40. Gros A, Turcotte S, Wunderlich JR, Ahmadzadeh M, Dudley ME, Rosenberg SA. Myeloid cells obtained from the blood but not from the tumor can suppress T-cell proliferation in patients with melanoma. Clin Cancer Res. 2012;18(19):5212-5223.

41. Chikamatsu K, Sakakura K, Toyoda M, Takahashi K, Yamamoto T, Masuyama K. Immunosuppressive activity of $\mathrm{CD}_{14}{ }^{+} \mathrm{HLA}-\mathrm{DR}^{-}$cells in squamous cell carcinoma of the head and neck. Cancer Sci. 2012; 103(6):976-983.

42. Montero AJ, Diaz-Montero CM, Kyriakopoulos CE, Bronte V, Mandruzzato S. Myeloid-derived suppressor cells in cancer patients: a clinical perspective. J Immunother. 2012;35(2):107-115.

43. Fridlender ZG, et al. CCL2 blockade augments cancer immunotherapy. Cancer Res. 2010;70(1):109-118.

44. Nefedova Y, Nagaraj S, Rosenbauer A, Muro-Cacho C, Sebti SM, Gabrilovich DI. Regulation of dendritic cell differentiation and antitumor immune response in cancer by pharmacologic-selective inhibition of the janus-activated kinase 2 /signal transducers and activators of transcription 3 pathway. 
Cancer Res. 2005;65(20):9525-9235.

45. Bharti AC, Donato N, Aggarwal BB. Curcumin (diferuloylmethane) inhibits constitutive and IL-6-inducible STAT3 phosphorylation in human multiple myeloma cells. J Immunol. 2003;171(7):3863-3871.

46. Johnston PA, Grandis JR. STAT3 signaling: anticancer strategies and challenges. Mol Interv. 2011; 11(1):18-26.

47. Sharafinski ME, Ferris RL, Ferrone S, Grandis JR. Epidermal growth factor receptor targeted therapy of squamous cell carcinoma of the head and neck. Head Neck. 2010;32(10):1412-1421.

48. Argiris A, et al. Serum biomarkers as potential predictors of antitumor activity of cetuximab-con- taining therapy for locally advanced head and neck cancer. Oral Oncol. 2011;47(10):961-966.

49. Kumar B, et al. EGFR, p16, HPV Titer, Bcl-xL and p53, sex, and smoking as indicators of response to therapy and survival in oropharyngeal cancer. J Clin Oncol. 2008;26(19):3128-3137.

50. Wang L, Yi T, Kortylewski M, Pardoll DM, Zeng D, Yu H. IL-17 can promote tumor growth through an IL-6-Stat3 signaling pathway. J Exp Med. 2009; 206(7):1457-1464.

51. Chen CC, et al. Significance of interleukin-6 signaling in the resistance of pharyngeal cancer to irradiation and the epidermal growth factor receptor inhibitor. Int J Radiat Oncol Biol Pbys. 2010;76(4):1214-1224.
52. Tu SP, et al. Curcumin induces the differentiation of myeloid-derived suppressor cells and inhibits their interaction with cancer cells and related tumor growth. Cancer Prev Res (Phila). 2012;5(2):205-215.

53. Albesiano E, et al. Immunologic consequences of signal transducers and activators of transcription 3 activation in human squamous cell carcinoma. Cancer Res. 2010;70(16):6467-6476.

54. Dang EV, et al. Control of T(H)17/T(reg) balance by hypoxia-inducible factor 1. Cell. 2011;146(5):772-784.

55. Corraliza IM, Campo ML, Soler G, Modolell M. Determination of arginase activity in macrophages: a micromethod. J Immunol Methods. 1994; 174(1-2):231-235. 